\title{
PDLA a potential new potent topical analgesic: a case report
}

\author{
This article was published in the following Dove Press journal: \\ Local and Regional Anesthesia \\ 24 October 2014 \\ Number of times this article has been viewed
}

\author{
Joel S Goldberg ${ }^{1,2}$ \\ 'Durham Veterans Affairs Medical \\ Center, ${ }^{2}$ Duke University School of \\ Medicine, Durham, NC, USA
}

Correspondence: Joel S Goldberg Durham Veterans Affairs Medical Center, 508 Fulton Street, Durham, NC, 27705, USA

Email joel.goldberg2@va.gov

\begin{abstract}
Polymer D-lactic acid (PDLA) is a hydrogel that has been shown to sequester L-lactate (lactate). This reaction is rapid, spontaneous, and non-enzymatic. Lactate has been shown to have many functions within the nervous system including its use as a secondary fuel to sustain neural activity and as a neuromodulator. In the central nervous system, lactate is produced in glial cells and shuttled to neurons to be used mostly as a fuel. Lactate dehydrogenase (LDH)1 is the predominant LDH isoform within neurons and unlike LDH5, it preferentially converts lactate to pyruvate which can be used to produce adenosine triphosphate (ATP). Considering that lactate is intimately involved in the sustenance of neural activity, PDLA was applied to an open wound and its effects were examined. The results showed that the application of PDLA induced topical analgesia. This may be the first report to demonstrate that sequestering lactate, a source of energy required to sustain the firing of action potentials in neurons, may produce analgesia.
\end{abstract}

Keywords: polymer D-lactic acid, topical analgesia, hydrogel

\section{Introduction}

In biological systems, L-lactate (lactate) is usually considered as a waste product of glycolysis. Recent evidence supports that lactate has an independent function in modulating neurotransmission and it serves as an important energy source for neurons. ${ }^{1,2}$ The brain contains considerable amounts of lactate that can be used as the substrate for glucose required for the production of adenosine triphosphate (ATP) when glucose is in short supply. ${ }^{3}$ Lactate is produced in glial cells and can be shuttled to neurons presumably for fuel. Lactate can be converted to pyruvate within neurons by lactate dehydrogenase (LDH)1. LDH1 is the predominant LDH isoform within neurons and it preferentially converts lactate to pyruvate unlike LDH5. ${ }^{4}$ Pyruvate can then be converted to acetyl-coA, which is utilized by neurons to produce ATP. It was postulated that if such a system of lactate utilization occurs in the central nervous system, a similar system may also exist in the peripheral nervous system.

In 1932, Feng used a sciatic nerve preparation to show that the addition of lactate improves a peripheral nerve's capacity to maintain sustained neural activity as measured by a galvanometer muscle deflection. ${ }^{5}$ He also showed that this sustained activity did not occur in nitrogenous environment confirming that the effects of lactate were through oxidation. ${ }^{5}$ Immke and McCleskey further verified the role of lactate as a neuromodulator by showing that lactic acid could enhance neural transmission of visceral afferents. ${ }^{1}$ 
In 2012, it was discovered that lactate binds to polymer D-lactic acid (PDLA) forming a presumed stereocomplex. This reaction has been described as sequestering or "trapping" lactate. ${ }^{6}$ The evidence that this reaction occurs was demonstrated by two separate enzymatic methods and highperformance liquid chromatography. ${ }^{6}$ The reaction is rapid, spontaneous, and non-enzymatic at $37^{\circ} \mathrm{C}$. Sequestering lactate with PDLA is an extraordinarily versatile chemical reaction, which has many potential medical and non-medical uses. This case report shows that topical application of PDLA in the form of a hydrogel could produce analgesia by sequestering lactate, which is the energy source required by nociceptors to sustain the propagation of action potentials.

\section{Methods}

Pandey and Aswath first described the synthesis of poly-Llactic acid (PLLA) from L-lactic acid in a home microwave. ${ }^{7}$ The method to produce PDLA from D-lactic acid follows the same procedures as those used to produce PLLA from L-lactic acid. Beginning with low power and short time intervals, D-lactic acid was dehydrated and polymerized to form PDLA. D-lactic acid was then microwaved until there was approximately $50 \%$ loss of weight secondary to dehydration and esterification. In addition, the PDLA dimer $(n=2)$ and tetramer $(n=4)$ were synthesized at the Duke University Small Molecule Synthesis Facility by Dr David M Gooden and tested for enzymatic activity using Accuvin test strips (Accuvin LLC, St Napa, CA, USA). Fifty microliter aliquots of $180 \mathrm{mg} / \mathrm{L}$ lactic acid were incubated with $10 \mathrm{mg}$ of PDLA,

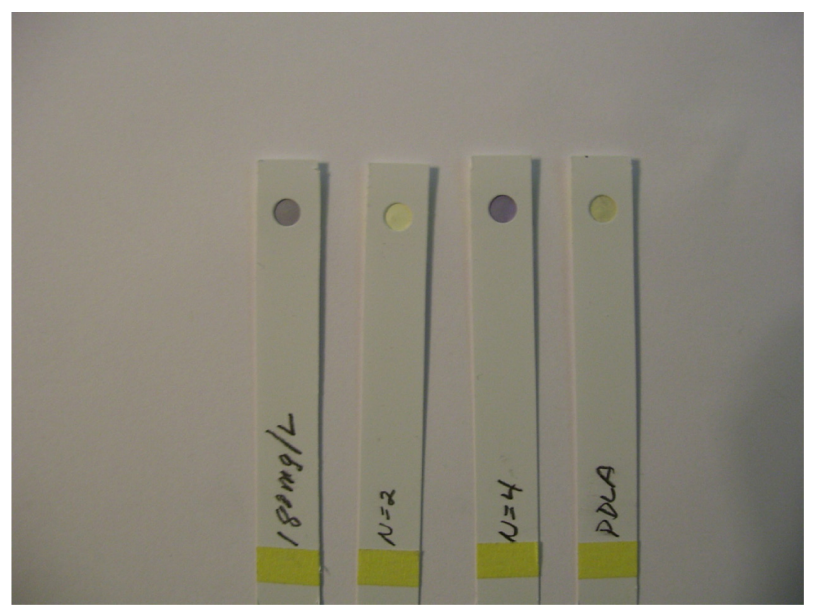

Figure I Enzymatic testing for lactic acid using Accuvin test strips.

Notes: Accuvin test strips from Accuvin LLC, St Napa, CA, USA. 180 mg/L lactic acid tested positive for lactic acid. $180 \mathrm{mg} / \mathrm{L}$ lactic acid and PDLA dimer $(\mathrm{N}=2)$ tested negative for lactic acid. $180 \mathrm{mg} / \mathrm{L}$ lactic acid and PDLA tetramer $(\mathrm{N}=4)$ tested positive for lactic acid. $180 \mathrm{mg} / \mathrm{L}$ lactic acid and PDLA oligomer mixture tested negative for lactic acid.

Abbreviation: PDLA, polymer D-lactic acid.
PDLA dimer $(n=2)$, and PDLA tetramer $(n=4)$ in three separate vials for 5 minutes at $37^{\circ} \mathrm{C}$, and then a $20 \mu \mathrm{L}$ aliquot from each vial was applied to each test strip (Figure 1).

We requested permission to apply PDLA in our patient from the United States Food and Drug Administration's Division of Anesthesia, Analgesia, and Addiction Products for emergency use. However, their response was that PDLA prepared in a home microwave is considered as a form of alternative medicine and hence, it is not within their jurisdiction to grant consent and permission may be granted directly from the patient for such use of alternative medicine. The patient gave permission to use PDLA for treatment of his wound.

\section{Results}

The test strips were activated by the control $(180 \mathrm{mg} / \mathrm{L}$ of lactic acid), and the solution of $180 \mathrm{mg} / \mathrm{L}$ lactic acid containing the PDLA tetramer $(n=4)$. The test strips were not activated by $180 \mathrm{mg} / \mathrm{L}$ solution of lactic acid containing the PDLA dimer $(n=2)$ nor the $180 \mathrm{mg} / \mathrm{L}$ of lactic acid containing PDLA, which consisted of a mixture of oligomers formed during the synthesis in the microwave (Figure 1).

\section{Case report}

The case patient had a $0.5 \times 1.5 \mathrm{~cm}$ skin lesion in the left index finger with an approximate depth of $1.5 \mathrm{~mm}$. The lesion produced vigorous bleeding and pain. Application of approximately $10 \mathrm{mg}$ of PDLA in the form of a hydrogel to the lesion produced an intense burning pain that lasted for approximately 30 seconds until there was complete analgesia without complete loss of sensation (numbness). The analgesia lasted for 24 hours until PDLA was removed by water. After washing the wound with water, the pain returned and additional PDLA was applied, which produced continuous analgesia until the lesion was completely healed after 10 days.

\section{Discussion}

The test strip experiment confirms that the PDLA dimer $(n=2)$ sequesters lactate and is a likely component of the PDLA microwave mixture. It also confirms that the PDLA tetramer $(n=4)$ does not sequester lactate. The case report demonstrates that the PDLA mixture prepared in a microwave has analgesic properties when applied to a patient's open wound.

Topical analgesics and local anesthetics employ a variety of mechanisms to produce analgesia. Topical analgesics can produce analgesia either by stimulation of A-beta fibers (eg, through application of counter irritants or transcutaneous 
electrical stimulation) or by some forms of massage involving presumed inhibition of nociception at the dorsal horn. Topical local anesthetics in current clinical use interfere with conductance of sodium by blocking the propagation of action potentials. Topical capsaicin deletes substance $\mathrm{P}$, which is a known excitatory neurotransmitter. This case report shows that application of PDLA hydrogel produces analgesia presumably by sequestration of lactate depleting the energy source required by nociceptors to sustain neural activity. This mechanism is supported by prior work demonstrating the importance of lactate in the sustenance of action potentials. ${ }^{1,5}$ Since the analgesic effects could be reversed by washing the wound with water, it suggests that such effects are not secondary to neurolysis. Also, PDLA used in this case report exists as a hydrogel and hence, it may serve as a protective barrier for healing.

Depleting the energy source required by nerves to propagate action potentials may be a new mechanism to produce analgesia. If the active oligomers of PDLA could be introduced into the central nervous system, the ability of PDLA to sequester lactate may be used as a potential mechanism for the treatment of posttraumatic stress disorder (PTSD) and mania. PTSD develops in susceptible individuals who undergo infusions of sodium lactate, and patients with mania are shown to have elevated lactate levels., ${ }^{8,9}$

Although the toxicity of PDLA is not known, PDLA is a known component in cardiac stents, biodelivery systems, and wound coverings. ${ }^{10,11}$ It also has been used as part of drug delivery systems.

\section{Conclusion}

Topical application of a PDLA hydrogel induced analgesia in the patient with an open wound. PDLA may have two beneficial effects for wound healing: 1) providing a protective barrier as a hydrogel; and 2) serving as an analgesic by sequestering lactate, a primary fuel source required for the generation of nociceptive action potentials.

\section{Acknowledgment}

The author would like to thank Dr David M Gooden and staff of the Small Molecule Synthesis Facility at Duke University, who were commissioned to synthesize the PDLA dimer and tetramer.

\section{Disclosure}

The author reports no conflicts of interest in this work.

\section{References}

1. Immke DC, McCleskey EW. Lactate enhances the acid-sensing Na+ channel on ischemia-sensing neurons. Nat Neurosci. 2001;4(9): 869-870.

2. Philp A, Macdonald AL, Watt PW. Lactate - a signal coordinating cell and systemic function. $J$ Exp Biol. 2005;208(Pt 24):4561-4575.

3. Schousboe A, Westergaard N, Waagepetersen HS, Larsson OM, Bakken IJ, Sonnewald U. Trafficking between glia and neurons of TCA cycle intermediates and related metabolites. Glia. 1997;21(1):99-105.

4. Bittar PG, Charnay Y, Pellerin L, Bouras C, Magistretti PJ. Selective distribution of lactate dehydrogenase isoenzymes in neurons and astrocytes of human brain. J Cereb Blood Flow Metab. 1996;16(6):1079-1089.

5. Feng TP. The role of lactic acid in nerve activity. J Phsyiol. 1932;76(4): 477-486.

6. Goldberg JS, Weinberg JB, inventors. Use of polymer D-lactic acid (PDLA) or equivalents thereof to inhibit growth of cancer cells and diagnose cancers. United States patent US 20120195850. August 2, 2012.

7. Pandey A, Aswath PB. Microwave synthesis of poly(L-lactic acid). J Biomater Sci Polym Ed. 2009;20(1):33-48.

8. Jensen CF, Keller TW, Peskind ER, et al. Behavioral and neuroendocrine responses to sodium lactate infusion in subjects with posttraumatic stress disorder. Am J Psychiatry. 1997;154(2):266-268.

9. Regenold WT, Phatak P, Marano CM, Sassan A, Conley RR, Kling MA Elevated cerebrospinal fluid lactate concentrations in patients with bipolar disorder and schizophrenia: implications for the mitochondrial dysfunction hypothesis. Biol Psychiatry. 2009;65(6):489-494.

10. Tamai H, Igaki K, Kyo E, et al. Initial and 6-month results of biodegradable poly-l-lactic acid coronary stents in humans. Circulation. 2000;102(4):399-404.

11. Panyam J, Labhasetwar V. Targeting intracellular targets. Curr Drug Deliv. 2004;1(3):235-247.

\section{Publish your work in this journal}

Local and Regional Anesthesia is an international, peer-reviewed, open access journal publishing on the development, pharmacology, delivery and targeting and clinical use of local and regional anesthetics and analgesics. The journal welcomes submitted papers covering original research, basic science, clinical studies, reviews \& evaluations,

\section{Dovepress}

guidelines, expert opinion and commentary, case reports and extended reports. The manuscript management system is completely online and includes a very quick and fair peer-review system, which is all easy to use. Visit http://www.dovepress.com/testimonials.php to read real quotes from published authors. 